\title{
Science unfolding in time: a situated protein folding landscape in retrospect
}

\author{
Alexander Powell \\ ap.cybercraft@googlemail.com \\ ORCiD: https://orcid.org/0000-0001-6911-1890
}

5 September 2019

(Version 1: 30 August 2019)

\begin{abstract}
A previously unpublished synthesis of ideas about protein structure and folding is presented. Incorporating a number of theoretical speculations and proposals for research, it sheds light on what researchers knew at a specific point in time and how they were thinking about a fundamental but recalcitrant biological problem. Additional context and insight come from graphical representations of the occurrence over time of key terms in the literature relating to protein structure and its wider scientific context.
\end{abstract}

Keywords: protein folding, protein structure, molecular biophysics, molecular modeling, structural biology, bioinformatics, history of science

\section{Introductory note}

In August 1989, when I wrote the previously unpublished essay presented below, I was ten months into a postgraduate research studentship in the antibody engineering and modelling group of Tony Rees at the Laboratory of Molecular Biophysics (LMB) in Oxford. Not long afterwards I moved a short distance up South Parks Road to Chris Dobson's NMR group in the Inorganic Chemistry Laboratory, following Tony's appointment as Professor of Biochemistry at Bath University.

Much has changed in thirty years. In 1989 the LMB was still part of the Department of Zoology, whereas now molecular biophysics persists as a set of service functions within the Department of Biochemistry [1]. Little more than a glimmer was visible then of the potential significance of chaperones for protein folding in vivo [2,3], and the concept of moonlighting proteins still lay in the (not too distant) future [4]. Loops and disordered regions were recognised, and indeed were often treated effectively as a species of secondary structure, but the idea that some proteins might, as a matter of functional necessity, be intrinsically disordered was unknown [5,6]. 2-D NMR techniques were developing apace, with Wüthrich's NMR of Proteins and Nucleic Acids the practitioner's bible [7], and 3-D NMR was just entering the scene [8]. Molecular dynamics (MD) simulation was on its way to becoming part of the 
structural biologist's standard arsenal [9], though it taxed the available computational resources heavily [10]; McCammon and Harvey's Molecular Dynamics of Proteins and Nucleic Acids had recently appeared [11]. Crystallographic refinement could make use of simulated annealing [12] and MD via Axel Brünger's X-PLOR suite [13,14]. Graphics facilities at Oxford included the ubiquitous Frodo software [15] running on Evans \& Sutherland workstations, as well as Biosym's Insight / Discover suite running on Silicon Graphics hardware.

My principal original motivation for writing the essay was to straighten out my thoughts about a topic that had captivated me. Building on what I had learnt previously as a biochemistry undergraduate at Bristol University, especially in my final year project supervised by Hilary Muirhead, my knowledge of protein structure and the techniques associated with its study had deepened considerably since the start of my postgraduate studentship in October 1988. I had been learning to apply NMR to peptides, though the method seemed arduous and somewhat unrewarding. I got on much better with computer modelling and graphics programming, and was especially excited by the idea of simulating protein folding and by Monte Carlo approaches to conformational sampling $[16,17,18,19]$. For reasons that the paper makes apparent, I was keen to investigate the representation levels and conformations of tripeptides in the Protein DataBank (PDB). Inspiration for this I suspect came at least in part from the structural surveys of Janet Thornton and colleagues at Birkbeck, which were known to me through published articles and also because Janet had lectured on her findings to an audience of Bristol biochemists when I was in my final year [20]. In fact I had begun tripeptide investigations prior to my switching research group. (A minor, and minor key, story could be told about how that switch came about and events subsequent to it, but here is not the place.)

Reading the essay now, I am struck by how far I went with the conformational space concept, construed in Cartesian terms. The image of stable conformations as low-energy caves, and discussion of the entropic costs of entering such caves, seem useful. I am reminded too of how dynamic were the terms in which proteins were then conceived: "... the native structure is a set of closely similar conformations (a conformational ensemble) ..."; "[an unfolded protein is] one in which each residue in the chain populates each phi/psi state with the frequency expected from Boltzmann population of the Ramachandran plot for that residue in the context of its neighbours on either side ...". The visual metaphor of folding as the descent of a potential energy funnel is, arguably, latent in the assertion that the "(e)arly phases of folding probably involve a multitude of pathways...", and robustness gets a mention in relation to the possibility of multiple folding pathways. The idea that an incompletely folded protein might act as a 'morphogenic surface' capable of inducing structure formation in peptides was one that I had presumably picked up somewhere rather than generated de novo. Perhaps the source was local, given Chris Dobson's work - which however I don't think had yet started in earnest in 1989 - on protein misfolding [21]. Or perhaps it stemmed from discussions about antibody-antigen interactions, and Janet Cheetham's NMR investigations of lysozyme peptide binding to the Gloop2 antibody, the structure of which Andrew Martin was modelling [22]. Somehow it was in the air, clearly. It is an idea that sits particularly well with the emergence of the concept of intrinsically disordered proteins and with contemporary trends in research in molecular recognition and signalling.

Amongst neglected and under-emphasized topics I would pick out structure prediction 
methods, electrostatics and water structure and behaviour for particular mention. In relation to the first of those, there is a reference to the secondary structure prediction method of Chou and Fasman, but there is nothing on homology-based modelling. The reason for this is that I was interested far more in ab initio methods and simulation-based approaches; modelling on the basis of sequence homology seemed more like an admission of defeat than a demonstration of knowledge. It is worth noting here that the threading approach had yet to be devised [23,24]. The absence of discussion of electrostatics is surprising, since I well remember James Warwicker visiting Oxford from Yale to give one of the regular LMB lunchtime talks, where he described the electrostatic field calculations he had been developing $[25,26]$. The idea that proteins might generate specific, functionally significant, electrostatic fields was very appealing to a computationally minded student of antibody-antigen recognition, concerned with understanding antibody specificity and the character of antigenic determinants. As for water, Thornton had analysed its structure around proteins in the PDB [27], but I may well have been guilty - and perhaps I was not alone in this - of to some extent neglecting water-protein interactions when thinking about protein folding and structure (albeit whilst acknowledging the critical overall importance of the hydrophobic effect, which the essay covers I think respectably enough). My inclination now would be to think in a rather more egalitarian way about polypeptide and water almost as a single molecular system and set of interactions [28,29]. One imagines solvent water molecules crawling over a polypeptide, hydrogen bonds forming and breaking as molecular dynamics, driven by thermal energy, constantly probes the overall potential energy space, with the polypeptide being reconfigured in the process.

From an autobiographical point of view the discussion of tripeptide analysis and the idea that statistical over- or under-representation of specific tripeptides might have particular importance for folding and structure is one that has a special significance. For this way of thinking, of seeking meaning in the relative occurrence frequencies of strings of tokens, with triplets of tokens having an appeal tied at that time to combinatoric factors and computational tractability, would later play an important part in work I did in the digital publishing domain. In 1992-3, for example, I explored the automatic identification of OCR errors by looking for linguistically improbable character trigrams, and a similar concern with relative occurrence frequencies informed work I carried out in the late 1990s on the extraction of key terms from academic journals content. The experience I gained in developing data structures to handle indexing operations then led naturally to my thinking about information retrieval, from which several software projects flowed. By way of further digression, another association of ideas now occurs to me which bridges the biological and linguistic domains. It concerns the relationship between token size and occurrence frequency. The essay notes the inverse correlation between the mass of an amino acid and the relative frequency with which it occurs, and in natural languages it is found that more common words tend to be shorter than rarer, more semantically specific, words. (This is sometimes seen as an instance of the Principle of Least Effort with which George Zipf is associated.)

Until recently, the essay has existed only as a print-out of a file originally authored in $T_{E} X$. A new digital version was obtained by scanning the manuscript and using optical character recognition (OCR) software to convert the resulting page images to text, followed 
by manual clean-up and formatting. Editorially I have confined myself for the most part to the correction of OCR errors and fixing a few obvious typos in the original manuscript. I have left unmodified the ampersands which I saw fit to use in place of 'and', as well as some unresolved figure references. Levinthal (1961) should presumably be Levinthal (1969). At one point a 'labreport' is cited. This would have been a reference to one of the LMB reports that Pam Bachelor, David Philip's personal assistant, compiled every year from reports submitted by the various research groups in the lab. I added reference citation cues in square brackets as I was writing, but never expanded them or compiled a bibliography. (I generously leave that as an exercise for the reader.)

In Appendix $A$ aspects of the protein folding landscape of thirty years ago are highlighted in a rather different, but complementary, way: graphically, as a series of Google Ngram Viewer outputs. These show how in the 1980s and 1990s certain terms came to be used more frequently as the contours of the field evolved, and bear witness to some of the points made above. This graphical approach reveals in a highly perspicuous manner key features of the overall context of the scientific snapshot from 1989 that the essay represents.

It may be that with the advent of 'deep learning' approaches and other techniques we stand on the threshold of a new era in our ability to predict protein structure [30,31]. Yet there is something slightly sobering in the fact that, despite now knowing the structures of over 400 times as many proteins as were known thirty years ago [32], and despite today's CPUs containing thousands of times as many transistors as they did then [33], our abilities to simulate folding and predict structure are not considerably better than they are [34]. A newly synthesized polypeptide - if it is not an IDP! - folds spontaneously, oblivious to how any other sequence, or indeed any other instance of the same sequence, has folded before. When, and perhaps only when, it becomes routinely possible to replicate the outcome of that process in silico for arbitrary amino acid sequences will we finally be able to say that the protein folding problem is solved. However, it remains up for debate, as a philosophical matter, whether such an assertion should be permitted if routinely successful in silico structure prediction were to proceed from a non-ab initio starting point. What confidence could we have in our understanding of the relevant fields and forces, for example, if we needed to add in 'something extra' to our prediction algorithms, in terms of knowledge (call it what you will) derived from large structural databases, when that is knowledge denied to the humble polypeptide as it comes off the ribosome?

\title{
The essay
}

\section{Caves \& Cores: Thinking about Protein Folding}

\author{
Alex Powell \\ August 7, 1989
}

An unfolded protein reaches the native conformation by tracing a path through conformational space (CS). Different points in this multi-dimensional space are associated with different potential energies, and are separated by energy barriers of 
varying magnitude. To get from point $A$ to point $B$ via a certain route requires that there is sufficient thermal energy available to surmount the energy barriers along that route. Some routes are blocked by barriers only traversable by covalent bond breakage, such as when one part of the chain passes through another part. But it may still be possible to get from A to B by going the 'long way around' in CS. The point to note is that close proximity in CS does not imply ready interconversion between two states.

Attempts to locate the lowest energy minima of a molecule in its conformational space by simple energy minimization algorithms are often thwarted because the molecule becomes trapped in local minima. This fact, together with the great complexity of polypeptide structures \& the large number of terms that contribute to the potential energy, suggests that potential energy surfaces in CS, if visualised in three dimensions, would appear extremely convoluted and irregular, with a good deal of fine structure. It is doubtful whether there are many extensive low many energy cavities \& manifolds in CS that are not frequently interrupted by significant energy barriers. This would imply the existence of well-defined folding pathways. Points in CS, besides having potential energy values, are associated with conformational probability values. The conformational probability integrated over the whole of CS has a value of unity. The relationship between energy \& relative occupancy is given by the Boltzmann formula $N_{\text {upper }} / N_{\text {lower }}=\exp (-\mathrm{d} E / \mathrm{k} T)$.

The native protein conformation does not necessarily lie at the global energy minimum, where this is taken to be the point in CS with the lowest potential energy. It is not inconceivable that the global minimum in fact lies in a region totally enclosed by energy barriers that can only be overcome by covalent bond breakage. Rather, the native conformation represents the point in CS with minimum potential energy that in addition is coupled to the denatured state by one or more pathways which do not contain any energy barriers that cannot be crossed using thermally available energy (several kT).

Folding pathways are likely to bifurcate, converge \& turn corners; the energies of neighbouring points in CS relative to a particular point dictate the statistical trajectory the chain will follow as the potential energy of the system decreases. Just as the course of a chemical reaction can be described graphically in terms of the way in which the

\section{< MS page 1 ends >}

energy varies with the 'reaction coordinate', so energy can be plotted against folding trajectory (progress through CS). The rates of catalysed reactions are enhanced because the activation energy is reduced; a reaction is broken down from a few large steps into a greater number of smaller ones, even though the initial \& final states are the same. Similarly, if there are two folding pathways between the same end points, most molecules will fold by way of the pathway with the lower conformational perturbation energy. 
Early phases of folding probably involve a multitude of pathways, populated by different numbers of molecules, while the latter stages may be more restricted to one or a few trajectories through CS. The first regions to fold are likely to be those with least resistance to conformational perturbation - regions where energy barriers are low. Conformational change may be impelled by predominantly local forces, or by more remote influences. Of course, if the forces are strong enough, higher energy barriers can be overcome. The existence of multiple folding pathways is not just a conjecture: elegant work on BPTI [ref. Creighton] testifies to their reality. It has been suggested that evolution favours multiple folding pathways, since this might be expected to lead to proteins robust to mutations that disrupt single pathways [ref. Harrison \& Durbin (1985)].

Folding experiments are performed in vitro, which is to say under a set of conditions very different from those under which a protein folds when first formed in vivo. Are conclusions reached in one domain transferable to the other without loss of validity? There is persuasive evidence to suggest that they are. Early experiments by Anfinsen showed that ribonuclease that is chemically denatured in vitro can spontaneously refold to regain the active conformation when the denaturing conditions are relaxed [ref. Anfinsen]. Subsequent work has shown that many other proteins can be reversibly denatured in vitro without loss of activity. This can be taken to suggest either that the protein folding timescale is much shorter than the protein synthesis timescale or that the $\mathrm{N}$-terminal region folds earlier than the $\mathrm{C}$-terminal region. If the former supposition is correct, \& the kinetics of synthetic addition of an amino acid on the ribosome were sufficiently sluggish, say for reasons of amino acid scarcity, then folding might proceed abnormally because synthesis would be incomplete over the time-window of folding. (Such an exigency would force the fulfilment of the second alternative.)

The potential energy of a given residue can be computed for different combinations of the dihedral angles phi \& psi, to generate a Ramachandran map [ref. Ramachandran (1969)]. This can be performed for a residue in the context of its N-terminal and Cterminal neighbours, giving a Ramachandran map for a specific tripeptide. A complete treatment would require the consideration of sidechain dihedrals chi(1)-chi(n), extending the map into higher dimensions. However, it is found experimentally that, despite the need for close packing of atoms [ref. Dill (1985)], sidechains tend to adopt certain preferred conformations [ref. Chothia (1984), labreport]. Early on in folding, the residue is likely to sample conformations in a manner consistent with the energetic topography of the map; thus it spends most of the time in those conformations with the lowest energy on the map, since local forces dominate in the unfolded chain. However, as folding progresses, more time might be spent in regions of the map which correspond to energetically less favourable conformations of the isolated tripeptide, as sequencedistant residues 


\section{< MS page 2 ends >}

come into close proximity to stabilise the locally unfavourable conformation. This is part of the basis of the observed cooperatively of protein folding. Hence the conformations of tripeptides as observed in X-ray crystal structures may be relatively uninformative about conformation-energy relationships in the isolated tripeptides. Nevertheless, the deviations are unlikely to be huge. For isolated tripeptides, Van der Waal's repulsion is the major delimiter of permissible conformations. The crystal conformations will lie within the tripeptide-allowed range, but with a distribution distorted by interactions with solvent molecules \& with residues some distance away in the sequence.

The native structure corresponds to a finite region of CS. It is not a point - the native structure is a set of closely similar conformations (a conformational ensemble) which dynamically interconvert by small \& larger scale motions [ref. McCammon \& Harvey]. The mean of these conformations is the point at the 'centre of gravity' of the native region of CS. This region can be thought of as a low energy 'cave' bounded by high energy barriers (except in a few directions). The native caves of different proteins may have different numbers of mouths, of varying sizes. Such differences will influence the thermal stability of the protein. There is a high entropic cost to be paid for entering a cave via a narrow entrance, but the probability of the native protein leaving the cave (unfolding) is lower. This only applies when all other things are equal; when the local energy barriers have the same magnitude, \& it is just the areas of the apertures that communicate between the native cave \& the rest of CS that differ.

The two conflicting requirements - for low entropic cost \& high thermal stability can be reconciled in various ways. The final energy barrier may be very high (but this slows folding), or the mean native conformation can be much more stable than other conformations (this seems rarely to be the case). Another solution is to make restraining covalent modifications once the the native cave is entered, e.g. sulphydryl pair oxidation to create a disulphide bridge. This has the effect of raising the height of the barriers around the native cave once it has been entered. Disulphide bridges only stabilize; they cannot render possible conformations that are thermally inaccessible. This is because for the bond to form, the two thiol groups must be in close apposition, \& they must reach this state by folding \& thermal energy alone. It would be interesting to ascertain whether a given disulphide is merely providing a 'safety net', preventing gross deformations of structure, or, at the other extreme, constraining the molecule to a conformation that would otherwise be much less stable. Crystallographic B-values of the bonding atoms \& their near neighbours could provide some information. The 'safety net' scenario implies the possibility of rather large motions about the the mean position, evidenced by moderately high B-values. The other case, where the disulphide is critical for stability, would be associated with low B-values. Molecular dynamics simulations using realistic potential expressions should also be able to place a given bond in a particular atomic environment accurately on a scale between the two extremes described. An 
initial examination of the refined crystal structure of hen egg white lysozyme shows no significant difference between the B-values of disulphide atoms \& other internal atoms.

The stability of a protein at a given point in CS is determined by a fine balance between a variety of competing factors. It is enhanced by the formation of hydrogen

\section{$<$ MS page 3 ends >}

bonds, by Van der Waal's attraction between neighbouring atoms \& favourable electrostatic interactions. These enthalpic terms are opposed by entropic effects; work must be done to restrict the motional freedom of a group. Entropy can favour folding, too. It is thought that the denatured, 'extended' polypeptide chain binds a substantial number of water molecules via its backbone amide groups. Upon folding, the waters are released into the bulk solvent with a corresponding entropy gain, which partially offsets the loss of entropy entailed by the condensation of the polypeptide chain. Perhaps the single most important determinant of stability is a phenomenon which is understood only in a qualitative way: the hydrophobic effect [ref. Tanford). This term denotes the tendency of 'oily' or hydrophobic groups to cluster together \& thereby become partitioned away from the aqueous environment that the protein molecule inhabits, to form an oily globule. The fact that hydrophobic groups lack any means of interacting favourably with polar water molecules leads to the creation of an ordered shell of water molecules around such groups. Ordered water structures (clathrate cages) have a lower entropy than the equivalent number of unrestricted water molecules; therefore it pays to minimize the total number of ordered waters. This is achieved by minimizing the surface:volume ratio of the oily globule, \& hence the clustering of hydrophobic moieties is energetically favoured over their dispersion throughout the solvent.

How can single amino acid changes disrupt folding? Suppose that the main folding pathway is preceded by two initial paths, Pathways $1 \& 2$. Normally, most of the molecules might follow Pathway 1. A mutation that blocks this pathway will force folding to proceed via Pathway 2, the slower one. In this instance only the kinetics of folding are affected, but no doubt more lethal substitutions are possible. Mutations can act at different points along the folding pathway(s); late acting ones might affect domain association, for example. Disruption of domain association may be functionally catastrophic, especially in enzymes in which the active site is at a domain interface (e.g. hexokinase, PGK), but if the individual domains fold independently \& more or less correctly then it seems infelicitous to call the mutation a folding disruptor.

Single domain folding is severely disrupted by buried mutations - the loss of a single methyl group from the hydrophobic core loses $50 \%$ of the free energy gain of folding [ref. Fersht - barnase]. Folding may proceed to a point at which elements of secondary structure are formed, \& these would then normally associate by clustering of hydrophobic faces. A mutation may prevent correct association by simple complementarity-breaking, or may preclude a relative rotation of two secondary 
structure elements that is required for the association to occur. (The latter effect observed in helix-turn-helix proteins: several residues at the 'elbow' between the the two helices are conserved, since mutations prevent the formation of the correct inter-helix geometry.) Many other possible folding disruptors can be envisaged. If elements of secondary structure form first, with association of the elements occurring in subsequent steps as is assumed in the 'framework' model of protein folding, one might expect folding to be inhibited at very high protein concentrations, due to competitive association of secondary structural units in different molecules. Indeed, folding of a protein is inhibited by the presence of fragments of the same protein in solution [ref.???]. This is not to say that such fragments adopt

\section{$<$ MS page 4 ends >}

a stable conformation in solution; the incompletely folded protein may act as a 'morphogenic surface' that induces structure in the peptide fragments. It is of interest to note in this regard that a short ex-lysozyme peptide (c.51-62), which adopts an extended beta conformation in the native protein environment, has been reported to form a short alpha-helix when bound to the peptide-specific T-cell receptor, even though in solution the peptide exhibits no detectable structure.

A reasonable hypothesis is that a fully denatured protein is one in which each residue in the chain populates each phi/psi state with the frequency expected from Boltzmann population of the Ramachandran plot for that residue in the context of its neighbours (i.e. the tripeptide map). An 'easy folder' might be a protein whose amino acid sequence is such that one of the highly populated denatured 'conformations' is quite close to that of the native ensemble. But because two points are distant in CS does not mean that folding is more difficult than when they are close, if they are connected by one or more pathways through CS that are unhindered by high energy barriers.

The denatured conformation represents a starting point for simulation of folding $\mathrm{ab}$ initio, when increased computer power makes this feasible. The set of denatured conformations places constraints on the possible subsequent trajectories through CS. It may be that certain tripeptides have numerous multiple energy minima, all of similar magnitude, distributed widely throughout phi/psi space. Others may be restricted to a few very pronounced minima. The remainder will fall somewhere between these two extremes. It seems likely that the 'extreme' tripeptides are of some importance in protein folding; they serve to delineate the most facile \& probable folding trajectories. There is a need to establish a library of phi/psi energy maps for each of the possible tripeptides.

There is little doubt that some short sequences are particularly important in generating structure [ref. Wright, Dyson \& Lerner]. Conversely, some sequences are inimical to the formation of certain kinds of structure, e.g. Pro cannot be accommodated within an alpha-helix without the introduction of a kink. Given a sequence-structure correspondence, can one reasonably expect some sequences to occur more or less often 
than if they were 'conformationally neutral', i.e. had no energetically preferred conformations? If proteins actually were composed of neutral peptides, then one would be back in the realm of Levinthal's notorious 'random search' (with allowances made for excluding chain crossings \& other topological impossibilities) [ref. Levinthal (1961)]. Of course, no amino acids really are conformationally neutral (although some are more adaptable, e.g. Gly, than others, e.g. Pro), \& hence neither are any peptides.

Side chain bulk is a major factor in determining conformational potential - large groups obviously preclude more conformations than smaller ones. Folding would seem to require both a limit on the number of possible conformations, to place the number of pathways within finite bounds \& thereby direct folding along particular lines, \& flexibility to keep down entropic costs. One wants multiple pathways (folding pathway degeneracy), for stability to mutations, between common end points. These are not necessarily incompatible demands: initial flexibility \& the corresponding degeneracy of the immediately possible folding trajectories can be seen to promote a rapid shrinkage in subsequently accessible conformational space.

\section{< MS page 5 ends >}

Sequence \& structural databases may be of use in identifying 'conformationally significant' sequences. It is well known that the statistical occurrences of individual amino acids in particular types of secondary structure can be used to predict the secondary structure of a novel polypeptide sequence with surprisingly high accuracy (up to $65 \%$ ) [ref. Chou \& Fasman]. It is interesting to note the pronounced inverse correlation between the mass of an amino acid \& the relative frequency with which it occurs (Fig.3). Glycine, the smallest amino acid, is the second most common, while tryptophan, the bulkiest, is the rarest. Glycine is, as expected, also found to adopt a greater range of conformations in crystal structures than any other residue (Fig.4). Lysine occurs with an infrequency that is inexplicable using steric or cost-of-synthesis arguments. This is undoubtedly due to the fact that, with a pKa near neutrality, it is chemically active in the physiological milieu, witness its key role in the catalytic mechanisms of many enzymes (e.g. the serine proteases). It is difficult to say whether the apparent mass-dependence of incidence frequency is a result of the conformational implications of side-chain bulk or is simply related to the energetic costs of synthesis of the amino acid. A subtle interplay frequency of factors may be at work, including thermodynamic dictates imposed by protein size, surface:volume ratios \& the hydrophobic effect [ref. Chothia (1984)].

Databases can be searched for all possible tripeptides. There are $8000\left(20^{*} 20^{*} 20\right)$ of these. In a database of $\mathrm{M}$ residues there are $(\mathrm{M}-2 \mathrm{~N})$ tripeptides, where $\mathrm{N}$ is the number of proteins. If we suppose that each of the 20 amino acids occurs equally often, we expect each possible tripeptide to occur $(\mathrm{M}-2 \mathrm{~N})^{*}\left((100 / 20)^{\wedge} 3\right)$ times. In a database of $1 \mathrm{~m}$ residues, $\&$ in which the average sequence length is 500 residues, each tripeptide is then expected to occur just over 124 times. If the individual probabilities of the amino acids making up 
a given tripeptide are p1, p2 \& p3 then the overall probability $\mathrm{P}$ of the tripeptide is obviously just $\mathrm{p} 1^{*} \mathrm{p} 2^{*} \mathrm{p} 3, \&$ the expected number of occurrences of the tripeptide in the database is $(\mathrm{M}-2 \mathrm{~N})^{*} \mathrm{P}$. In fact, the rarest amino acid occurs at a level of about $1.5 \%$. The least frequently-occurring tripeptide is then expected only just over 3 times. Clearly, to obtain statistically reliable figures demands a sizeable database. Current sequence databases contain approximately $3 \mathrm{~m}$ residues, so it should be feasible to derive some conclusions at least for tripeptides containing the more common amino acids.

If a particular tripeptide occurs in a database more or less frequently than would be expected on the basis of the occurrence-probabilities of the amino acids of which it is composed, then it may be justifiable to suppose that there is something peculiar about the properties of that tripeptide. What can be made of the 'over-occurrence' or 'underoccurrence' of a tripeptide? This is where structural \& functional data are invaluable. It may be that the tripeptide is conformationally unstable, but has a key role in the active site of a family of enzymes. It requires the rest of the enzyme to stabilize this critical region. This is analogous to the case of antibodies, in which a structurally overdetermined beta-barrel is sufficiently stable to bear any of a range of heterogeneous loops that confer functional specificity [ref. Rees]. The widespread occurrence of a peptide in a large number of seemingly unrelated proteins, \& in a diverse assortment of conformations, suggests that it is the flexibility \& conformational tolerance of the sequence that is being

\section{<MS page 6 ends >}

exploited. Knowing the occurrence-probabilities for individual residues, it is possible to propose 'improbable' sequences. If they occur frequently \& tend to be confined to specific families of related proteins it is not unreasonable to conclude that they are structurally significant sequences. More problematic is the precise role such sequences might play in dictating the formation of structure. Are they sites for the initiation of folding, or are they important in directing its later stages? Are they conserved because they drive the chain into a particular conformation, or in order to preserve complementarity between elements of secondary structure? Both theoretical \& experimental approaches have a part to play in answering these \& related questions.

It will not be long before it is possible to model accurately the dynamic behaviour of tripeptides \& larger sequences in solvent routinely. The expressions currently employed to calculate potential energies may need refinement. A $(12,6)$ Leonard-Jones potential is commonly used, despite the fact that 'there is plenty of evidence to show that $1 / \mathrm{R}^{\wedge} 12$ is a very poor representation of the repulsive potential, \& that an exponential form...is greatly superior' [Atkins]. Monte Carlo dynamics in torsional space, with energy minimisation of the solvent for each peptide conformation, affords a means of pseudo-uniformly sampling conformational space. In this way the potential energy through conformational space may be mapped. It should be possible to identify flexible sequences \& constrained ones, \& establish the relative populations of preferred 
conformations. Longer peptides, with correspondingly greater energetic contrast between least \& most favourable conformations, are amenable to experimental study by NMR, providing the basis for a dialectic between theory \& experiment. Shorter peptides usually sample a variety of conformations without adopting a dominantly stable structure. Even short peptides, however, may be sufficiently restricted as to spend a significant proportion of their time in one or a few well-defined conformations; the lifetimes of some of these conformations may be significant on the NMR timescale.

Already, much has been achieved in structure prediction using energetic computations involving empirically derived interatomic potentials. These methods can be termed 'dynamic' prediction techniques, since they involve successive atomic displacements to obtain the 'native' conformation. Therefore, they are folding simulations with empirically guided short-cuts. A 'buildup' procedure incorporating NMR data has been employed to model the structure of BPTI with reasonable accuracy [ref. Scheraga]. The sequence is broken down into di-, tri-, \& higher peptides which are then energy-minimized; the structure resulting from overlap of the lowest energy peptides is used as a basis for dynamics including experimental NOE restraints. The 'folding' of crambin has been modelled from the linear polypeptide by performing molecular dynamics with the sequential incorporation of NOE restraints, starting with just short-range NOEs \& gradually incorporating the longer range NOEs as 'folding' progresses [ref. Karplus]. NOEs must be used with caution, however; the relationship between atomic separation \& the intensity of the observed NOE is not always simple, especially where larger proteins are concerned [ref. Jardetsky]. The next stage is to dispense with experimental data altogether (although of course experimental results will be vital for establishing the existence of simulated folding pathways.) This truly would be 'knowledge-based' modelling. The further devel-

\section{$<$ MS page 7 ends >}

opment of massively parallel processing techniques could be the key to the realization of this goal.

Many researchers have sought to gain insights into the nature of the native state \& its relationship to other conformational states by classifying \& categorising the different types of structure observed in crystal structures. This is obviously essential for describing \& comparing proteins, but its utility as a route towards specifying the roles of individual residues in particular contexts is doubtful. Categorization is the grouping together of objects on the basis of the possession of common or unifying features. However, it may be that in considering protein folding, greater insight comes from a refusal to categorize, by abstaining from proposing universal pathways \& hierarchies of formation of structural motifs. The energetic topography of CS night be too complex for that. Perhaps every family of sequences is different, in terms of the number \& duration of folding intermediates, their modes of interconnection, their kinetics \& energetics. Unity then would stem from the fact that for all proteins, irrespective of sequence, the 
laws governing interactions between atoms are identical.

$<$ MS page 8 ends >

\section{Acknowledgements}

I am grateful to my Oxford supervisors Tony Rees and Chris Dobson for both the opportunities they provided and their support during a period that included the time when the essay featured here was written. Christ Church, Oxford, provided a home which at the time I was unable to appreciate adequately. I gratefully acknowledge the receipt of financial assistance, in research studentship form, from the Science and Engineering Research Council (SERC), as a major public funder of research in the UK was then known. Janet Cheetham showed me the NMR ropes, so to speak, and again I must express my deep gratitude to Janos Hajdu, Andrew Martin, and Dan Raleigh for their help, guidance and encouragement. I thank David Gregory for providing several useful references.

\section{References}

1. https://www.bioch.ox.ac.uk/molecular-biophysics (last accessed 1 September 2019)

2. Ellis, J. (1987) Proteins as molecular chaperones. Nature 328: 378-379.

3. Martin, J., et al. (1991) Chaperonin-mediated protein folding at the surface of groEL through a 'molten globule'-like intermediate. Nature 352: 36-42.

4. Jeffery, C.J. (1999) Moonlighting proteins. Trends in Biochemical Sciences 24(1); 8-11. DOI:https://doi.org/10.1016/S0968-0004(98)01335-8

5. Dyson, H.J. and Wright, P.E. (2002) Coupling of folding and binding for unstructured proteins. Curr. Opin. Struct. Biol. 12: 54-60. DOI:10.1016/S0959-440X(02)00289-0

6. Dunker, A.K., et al. (2002) Intrinsic disorder and protein function. Biochemistry 41(21): 6573-6582.

7. Wüthrich, K. (1986) NMR of Proteins and Nucleic Acids. Wiley-Interscience.

8. Oschkinat, H., et al. (1988) Three-dimensional NMR spectroscopy of a protein in solution. Nature 332: 374-376.

9. Elber, R. and Karplus, M. (1987) Multiple conformational states of proteins: a molecular dynamics analysis of myoglobin. Science 235(4786): 318-321.

10. Vendruscolo, M. and Dobson, C.M. (2010) Protein dynamics: Moore's Law in molecular biology. Current Biology 21(2): R68-R70. DOI: 10.1016/j.cub.2010.11.062.

11. McCammon, J.A. and Harvey, S.C. (1987) Dynamics of Proteins and Nucleic Acids. Cambridge: Cambridge University Press.

12. Kirkpatrick, S., Gelatt, C.D. and Vecchi, M.P. (1983) Optimization by Simulated Annealing. Science 220(4598): 671-680.

13. Brünger, A.T., Kuriyan, J. and Karplus, M. (1987) Crystallographic R factor refinement by molecular dynamics. Science 235(4787): 458-60. DOI:10.1126/science.235.4787.458.

14. Laboratory of Molecular Biophysics Annual Report 1988-89.

15. Jones, T.A . (1978) A Graphics Model Building and Refinement System for Macromolecules. Journal of Applied Crystallography 11: 268-272. DOI:10.1107/S0021889878013308.

16. Levitt, M. and Warshel, A. (1975) Computer simulation of protein folding. Nature 253(5494): 694698. 
17. Li, Z. and Scheraga, H.A. (1987) Monte Carlo-minimization approach to the multiple-minima problem in protein folding. Proc. Natl. Acad. Sci. USA 84(19): 6611-6615. DOI: 10.1073/pnas.84.19.6611.

18. Hansmann, U.H.E. and Okamoto, Y. (1999) New Monte Carlo algorithms for protein folding. Current Opinion in Structural Biology 9(2): 177-183.

19. Paquet, E. and Viktor, H.L. (2015) Molecular Dynamics, Monte Carlo Simulations and Langevin Dynamics: A Computational Review. BioMed Research International 2015, article ID 183918. DOI: http://dx.doi.org/10.1155/2015/183918.

20. Wilmot, C.M. and Thornton, J.M. (1988) Analysis and prediction of the different types of $\beta$-turn in proteins. Journal of Molecular Biology 203(1): 221-232. DOI: 10.1016/0022-2836(88)90103-9.

21. Dobson, C.M. (2003) Protein folding and misfolding. Nature 426(6968): 884-890.

22. Martin, A.C.R., Cheetham, J.C. and Rees, A.R. (1989) Modeling antibody hypervariable loops: a combined algorithm. Proc. Natl. Acad. Sci. USA 86(23): 9268-9272. DOI: 10.1073/pnas.86.23.9268.

23. Bowie, J.U., Lüthy, R. and Eisenberg, D. (1991) A method to identify protein sequences that fold into a known three-dimensional structure. Science 253(5016): 164-170. doi:10.1126/science.1853201.

24. Jones, D.T., Taylor, W.R. and Thornton, J.M. (1992) A new approach to protein fold recognition. Nature 358(6381): 86-89. doi:10.1038/358086a0.

25. Warwicker, J. and Watson, H.C. (1982) Calculation of the electric potential in the active site cleft due to alpha-helix dipoles. Journal of Molecular Biology 157(4): 671-679. DOI: 10.1016/00222836(82)90505-8.

26. Warwicker, J., Ollis, D., Richards, F.M. and Steitz, T.A. (1985) Electrostatic field of the large fragment of Escherichia coli DNA polymerase I. Journal of Molecular Biology 186(3): 645-649. DOI: 10.1016/0022-2836(85)90136-6.

27. Thanki, N., Thornton, J.M. and Goodfellow, J.M. (1988) Distributions of water around amino acid residues in proteins. Journal of Molecular Biology 202(3): 637-657. DOI: 10.1016/00222836(88)90292-6.

28. Bellissent-Funel, M.C., et al. (2016) Water Determines the Structure and Dynamics of Proteins. Chemical Reviews 116(13):7673-7697. DOI: 10.1021/acs.chemrev.5b00664.

29. Steinke, N., et al. (2018) Proline and water stabilization of a universal two-step folding mechanism for $\beta$-turn formation in solution. Journal of the American Society 140(23): 7301-7312. DOI: 10.1021/jacs.8b03643.

30. Hutson, M. (2019) Al protein-folding algorithms solve structures faster than ever, Nature, News section (22 July 2019). DOI: 10.1038/d41586-019-01357-6.

31. Dumé, B. (2019) Quantum approach reveals faster protein folding. Physics World, https://physicsworld.com/a/quantum-approach-reveals-faster-protein-folding/ (last accessed 30 August 2019)

32. https://www.rcsb.org/stats/growth/overall (last accessed 1 September 2019)

33. https://en.wikipedia.org/wiki/Moore\%27s law (last accessed 1 Septembr 2019)

34. Lowe, D. (2018) The Latest on Protein Folding. Science Translational Medicine / In the Pipeline blog. https://blogs.sciencemag.org/pipeline/archives/2018/12/03/the-latest-on-protein-folding (last accessed 30 August 2019) 


\section{Appendix A: Google ngram viewer outputs}

Figure A1: A general build-up

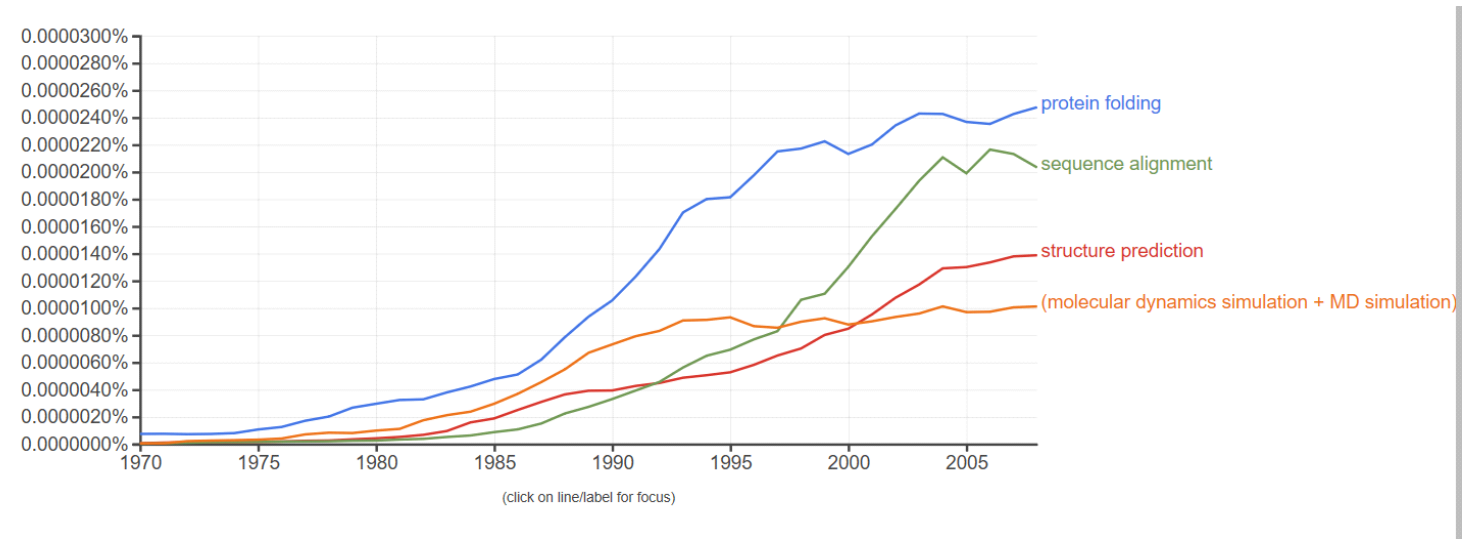

Figure A2: Disciplinary names in flux

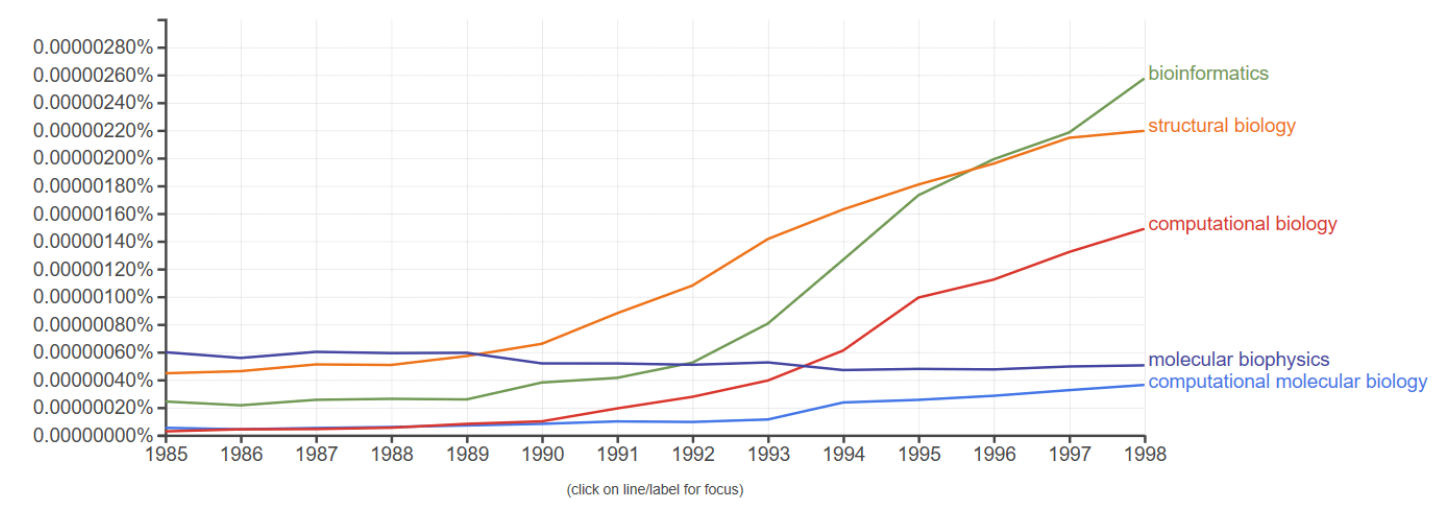

Figure A3: The protein engineering era

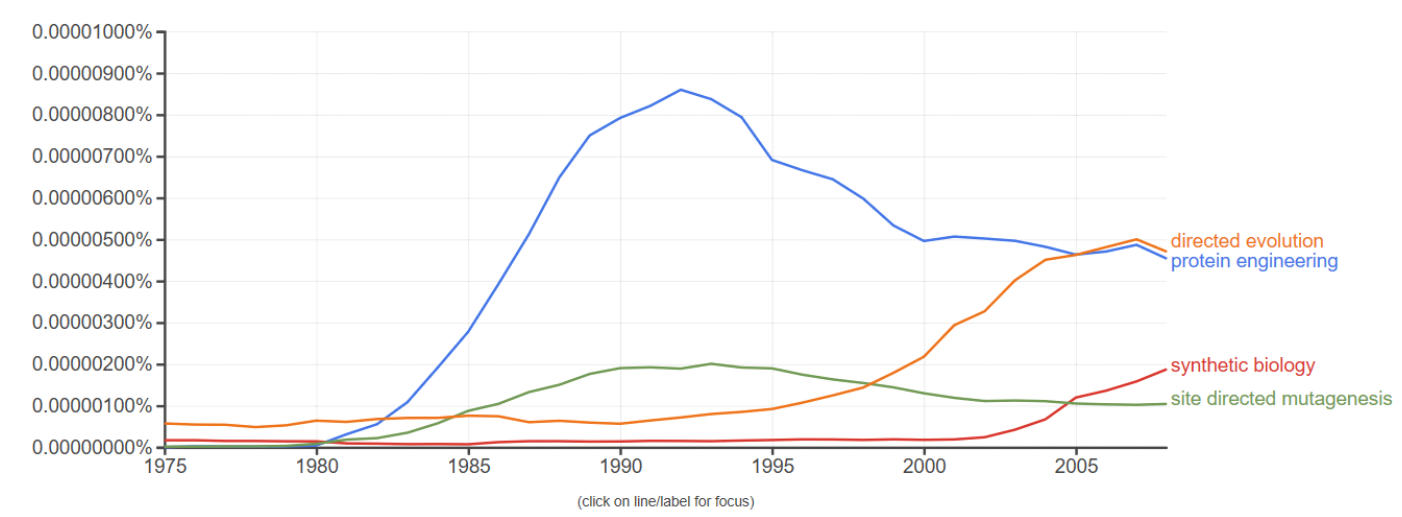


Figure A4: Chaperonins

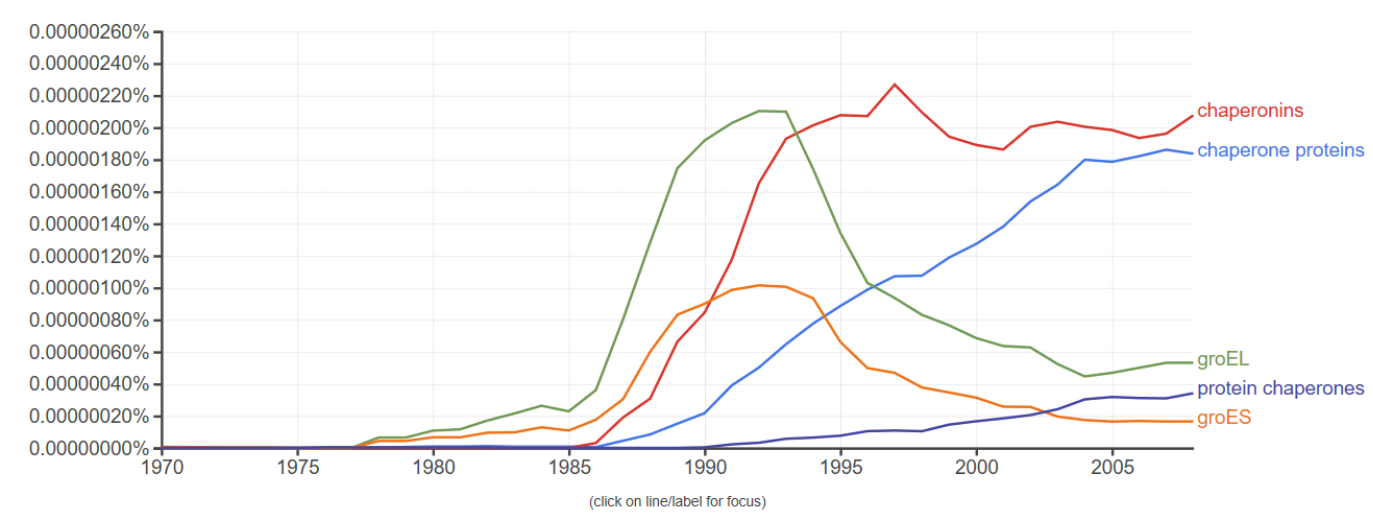

Figure A5: New classes of protein

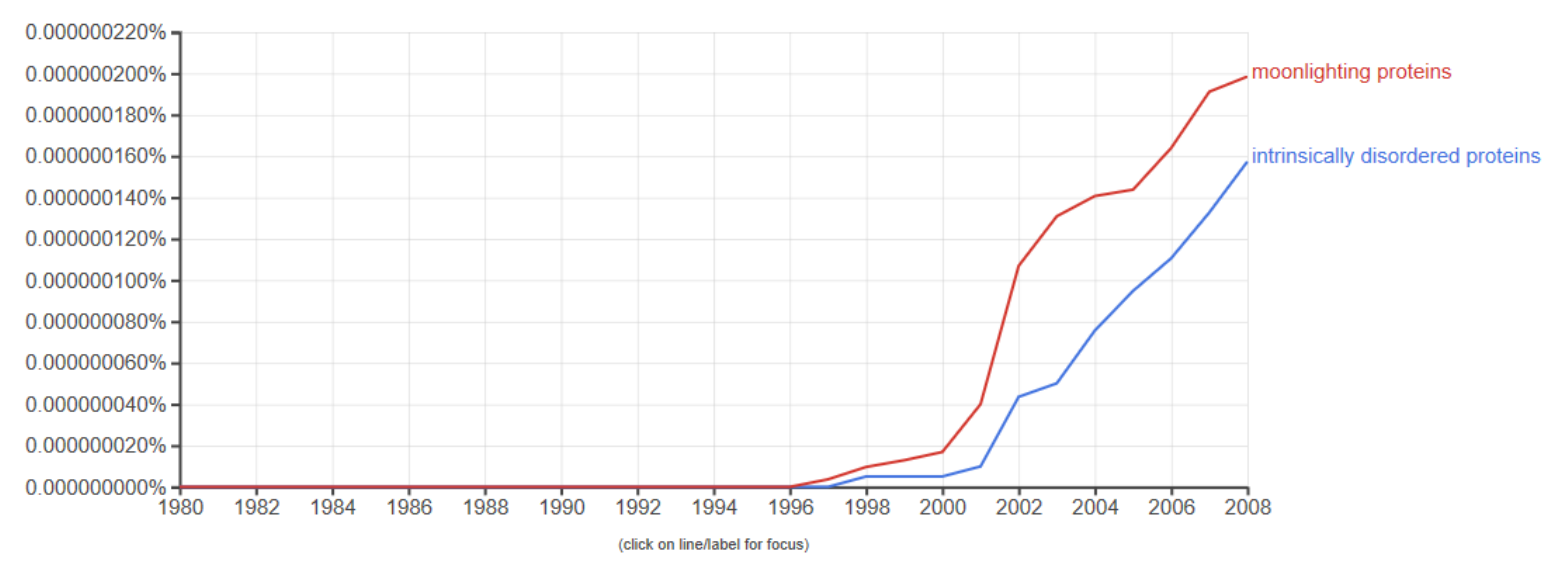

Figure A6: Laue crystallography

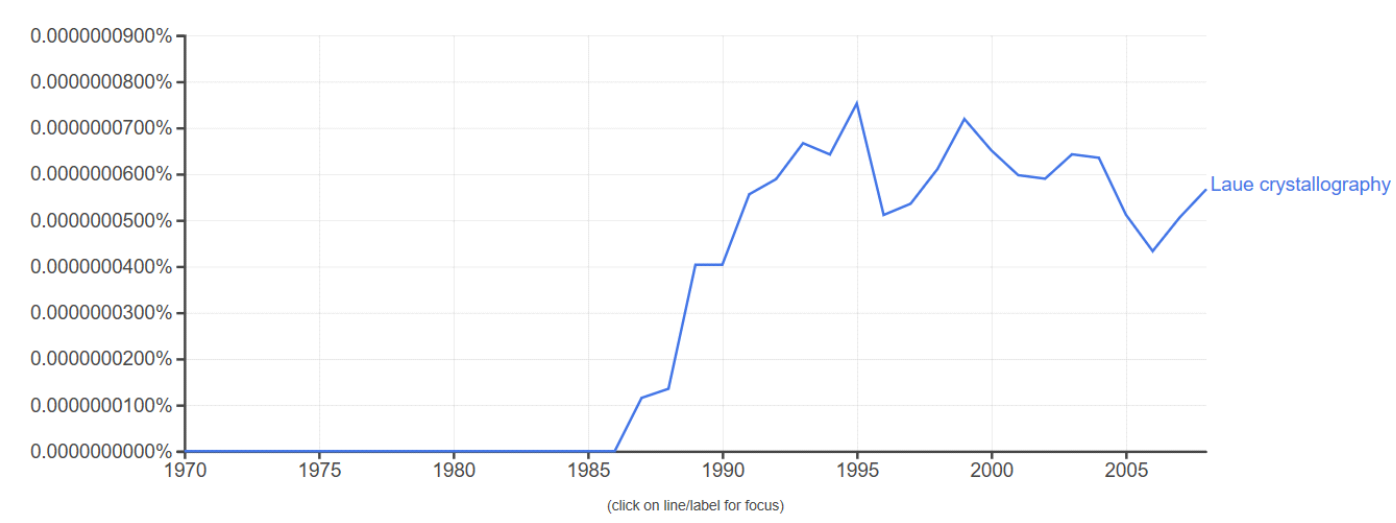


Figure A7: NMR evolves (I)

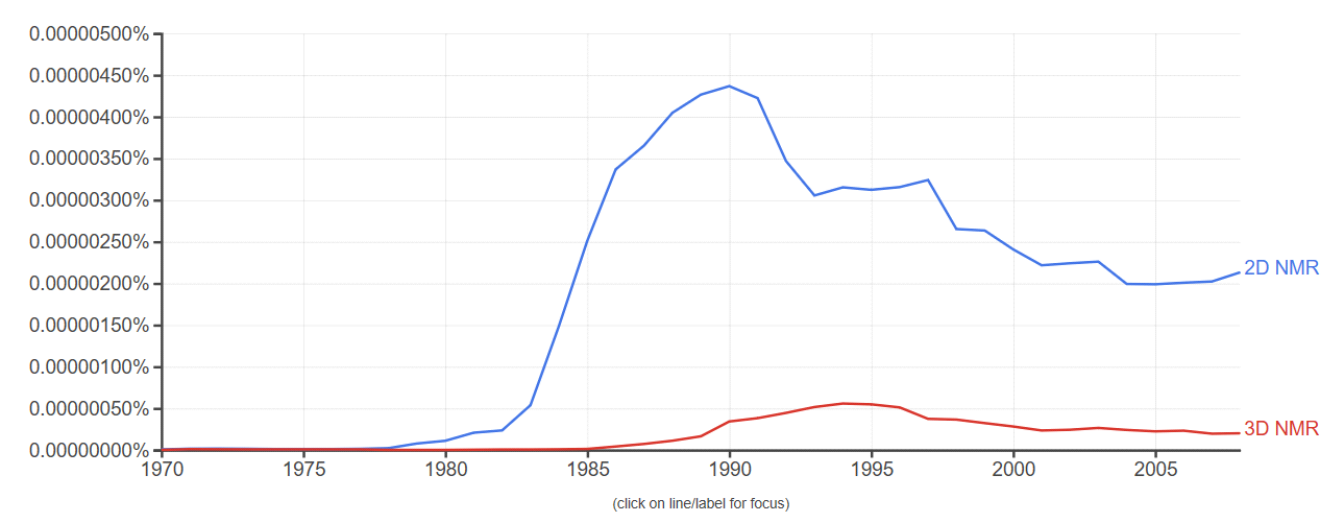

Figure A8: NMR evolves (II)

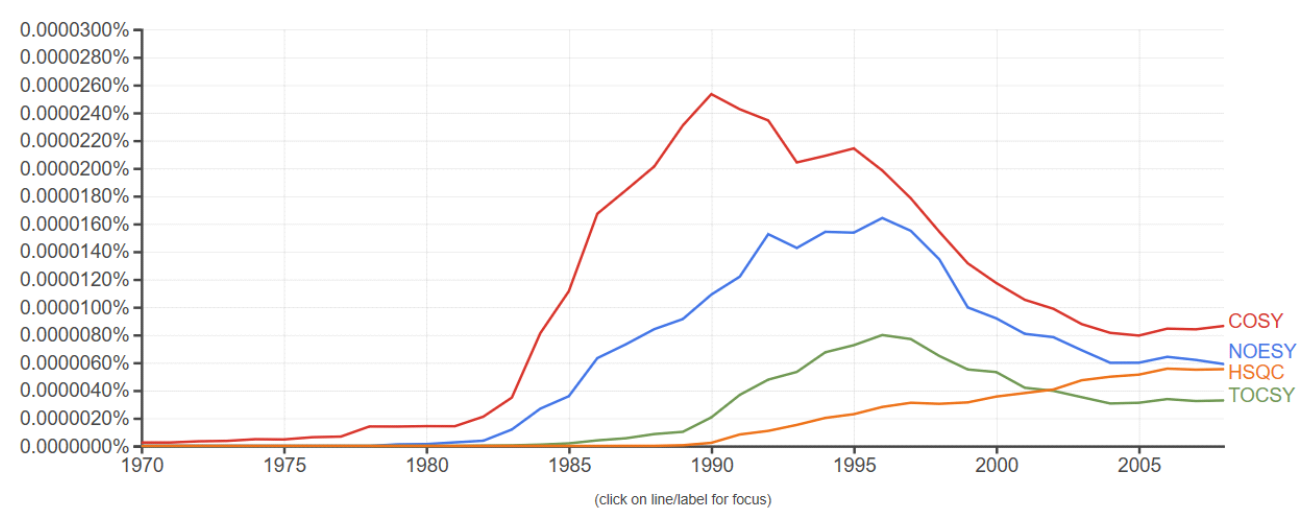

Figure A9: The hydrophobic effect

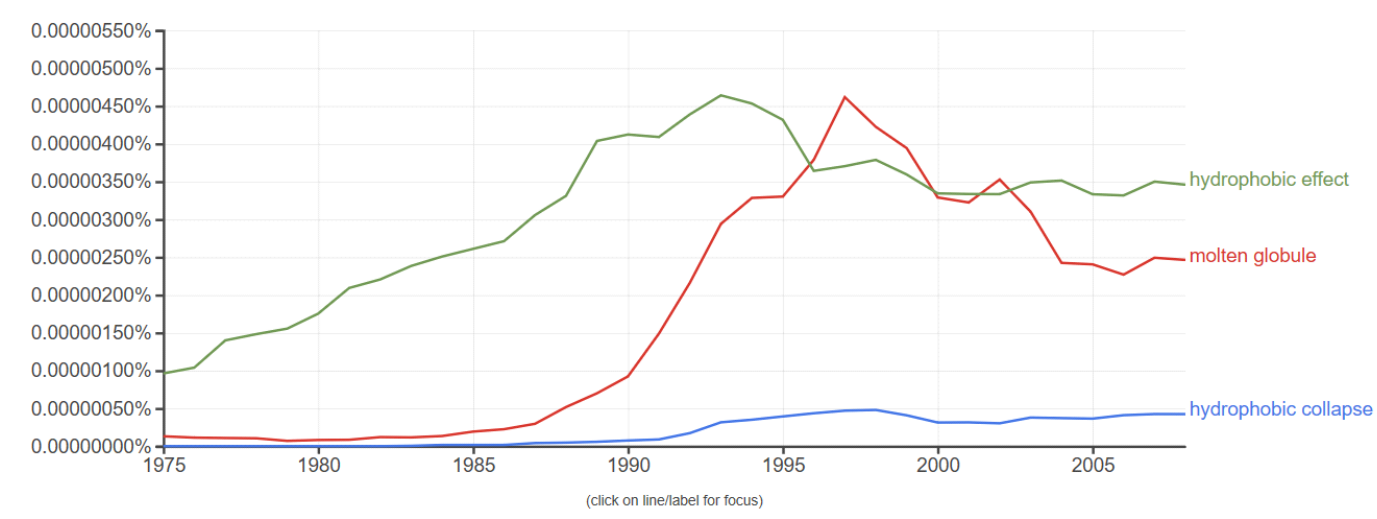


Figure A10: The paradox of the Levinthal paradox

It is curious that 'Levinthal paradox' should lag the other terms here so markedly.

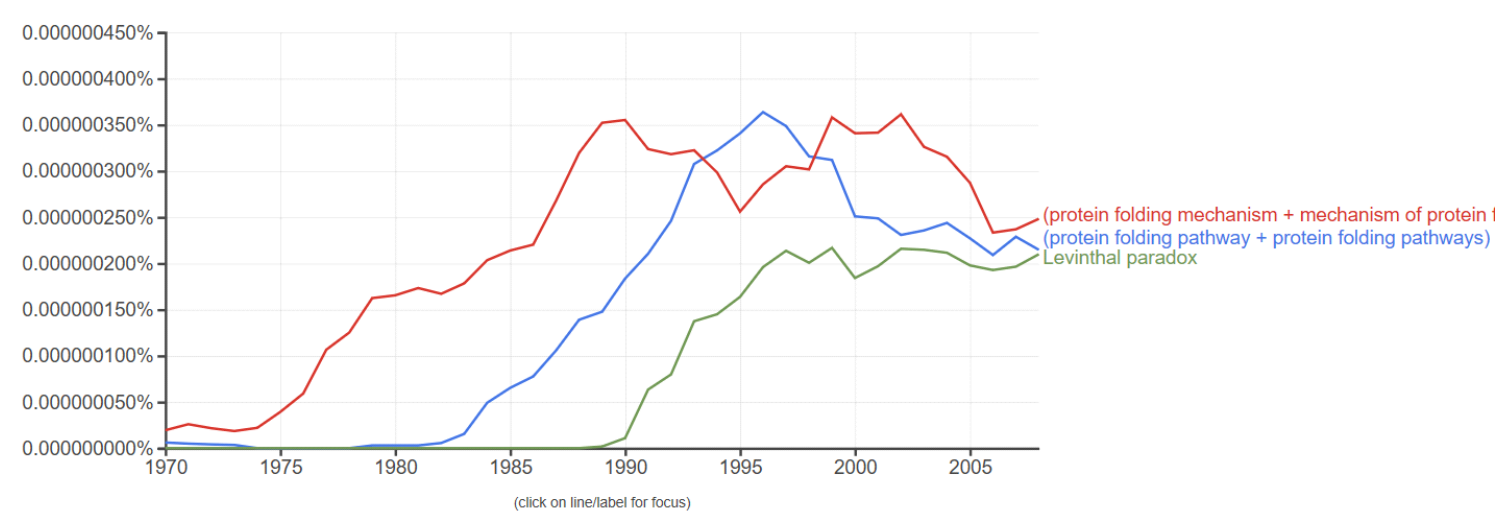

Figure A11: Molecular computing

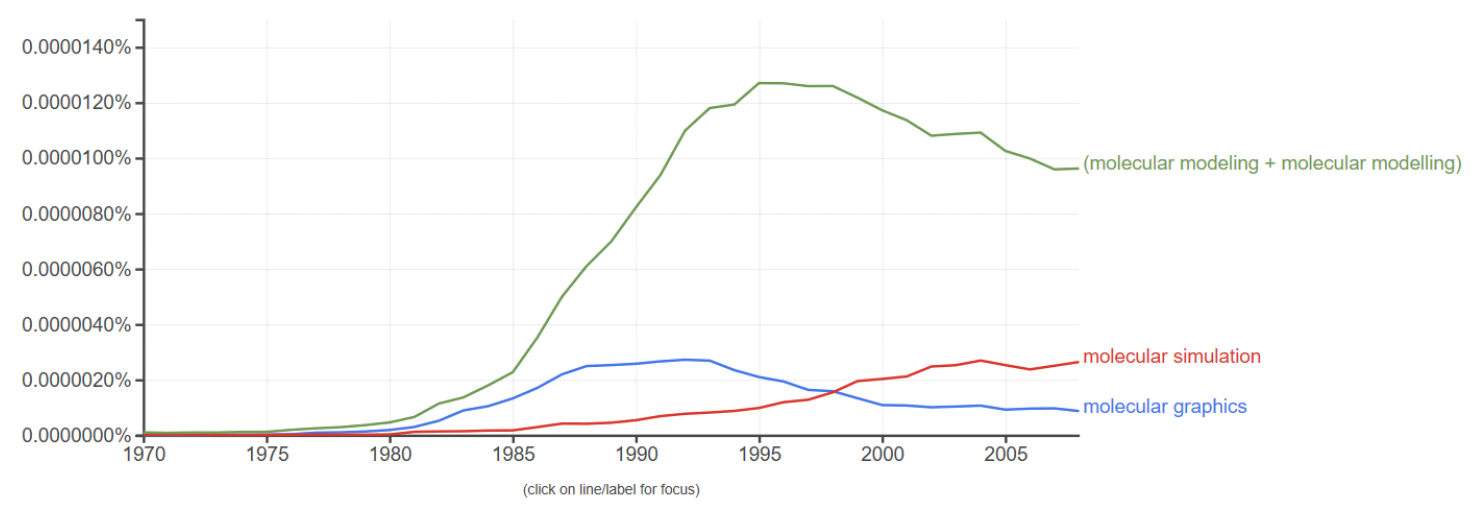

Figure A12: Threading and folds

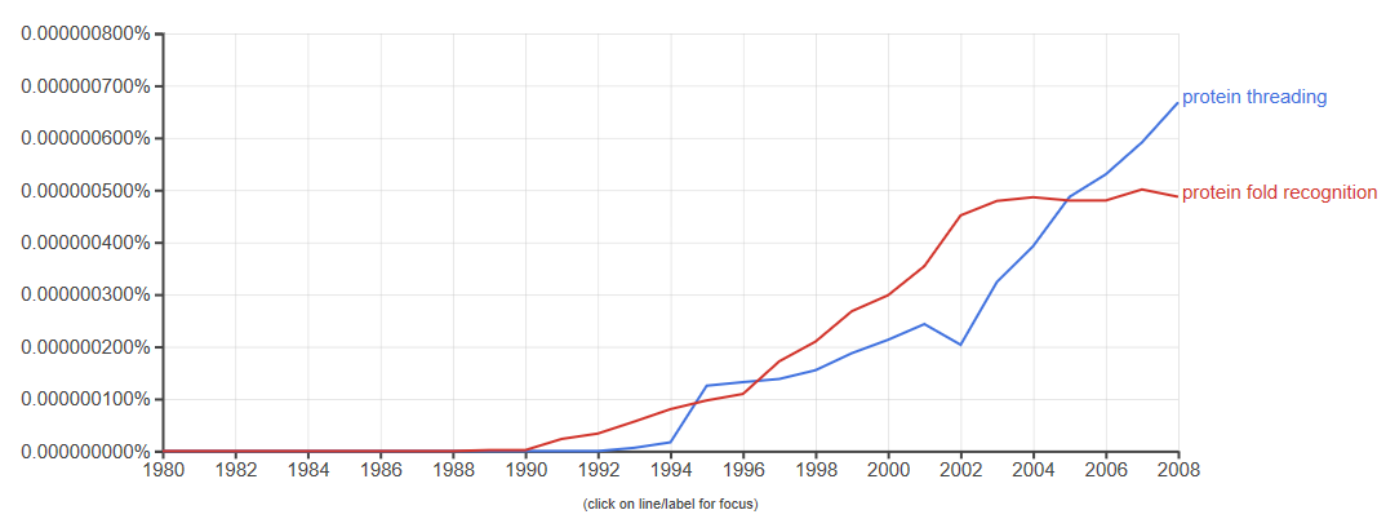


Figure A13: Computational approaches

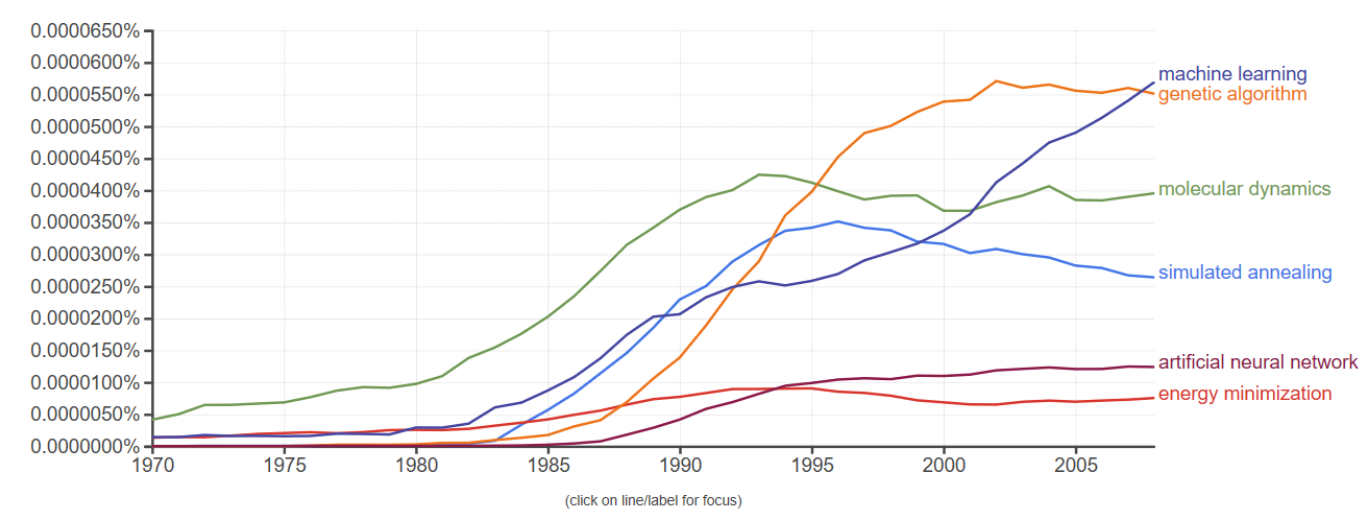

Figure A14: Computing hardware vendors

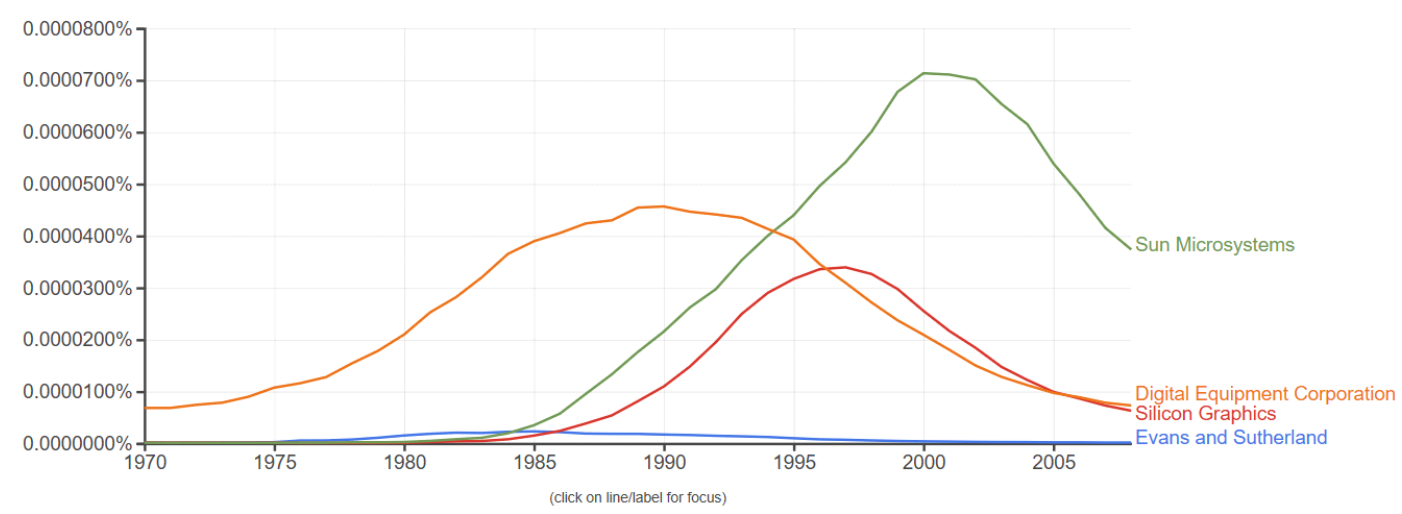

Figure A15: Programming languages

Note FORTRAN's long tail.

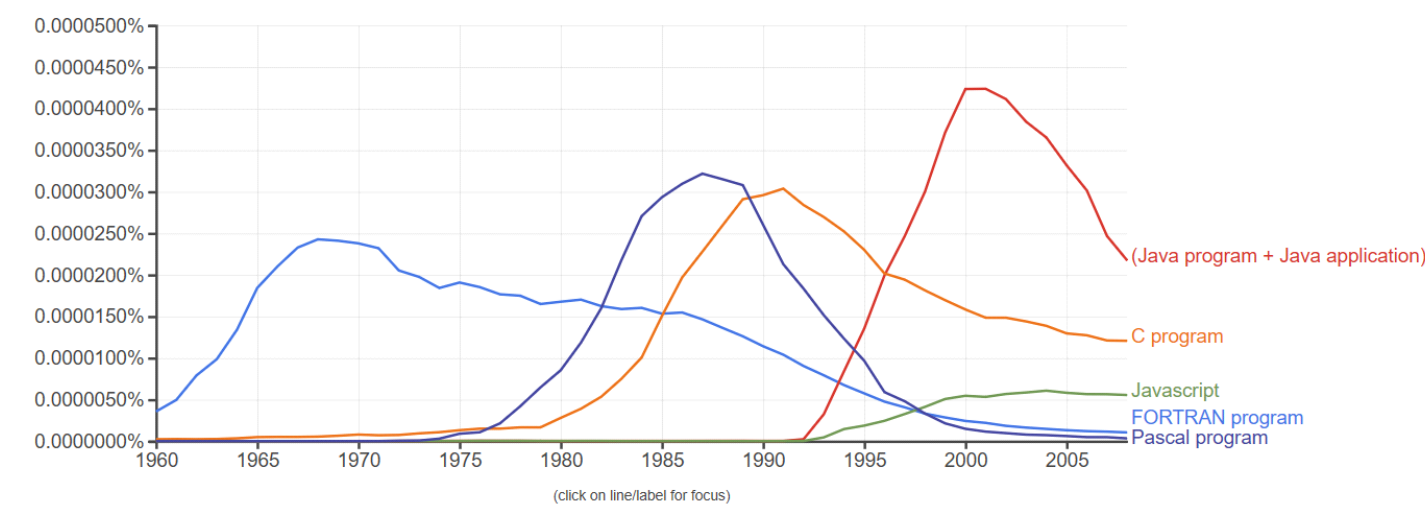

